\title{
Differential Heart Rate Reactivity and Recovery After Psychosocial Stress (TSST) in Healthy Children, Younger Adults, and Elderly Adults: The Impact of Age and Gender
}

\author{
Brigitte M. Kudielka, Angelika Buske-Kirschbaum, \\ Dirk H. Hellhammer, Clemens Kirschbaum
}

\begin{abstract}
In addition to numerous reports about psychophysiological stress responses to acute stressors, there are few data available on gender differences of stress-induced heart rate responses in multiple age groups applying the same psychological stressor. Second, the assessment of poststress recovery appears to be neglected in the empirical literature. For this study, data from 5 independent studies were reanalyzed to investigate the impact of age and gender on heart rate responses and poststress recovery to a standardized psychosocial stress task (Trier Social Stress Test; TSST) in 28 children, 34 younger adults, and 26 older adults.

As expected, prestressor baselines correlated significantly with chronological age $(r=-.27, p=.01)$. There was a marked age-related decrease in the heart rate stress response $(p=.0003)$ with children and younger adults showing significantly higher increases than elderly persons. The analysis of gender effects showed that girls had higher heart rate increases during the stress exposure than boys $(p=.03)$. In younger adults, stress responsivity was also higher in women $(p=.03)$. Peak heart rate responses were comparable in older men and women, with only men returning to prestressor baselines during the observation period.

In sum, this reanalysis revealed differential heart rate responses and recovery after exposition to the TSST in healthy children, younger adults, and elderly adults.
\end{abstract}

Key words: heart rate, age, gender, psychosocial stress, reactivity, recovery, Trier Social Stress Test (TSST)

A voluminous literature has accumulated over the last decades showing that a wide variety of different laboratory stressors lead to significant increases in heart rate (for reviews, see Haynes et al., 1991; Treiber et al., 2003; Matthews et al., 2003). In 1994, Seeman and Robbins introduced the term resilience to describe the overall pattern of physiological responses to challenge, encompassing the rate of initial response to challenge, the magnitude of the response, and the rate of recovery to the basal state. This theoretical concept points to the necessity to not only focus on the peak stress response but also on the recovery process after

Brigitte M. Kudielka, Department of Behavioral Sciences, Swiss Federal Institute of Technology (ETH), Zürich, Switzerland; Angelika Buske-Kirschbaum and Dirk H. Hellhammer, Department of Clinical and Theoretical Psychobiology, University of Trier, Trier, Germany; Clemens Kirschbaum, Department of Experimental Psychology, University of Düsseldorf, Düsseldorf, Germany

Correspondence concerning this article should be addressed to Brigitte M. Kudielka, Department of Behavioral Sciences, Swiss Federal Institute of Technology (ETH), TUR1, Turnerstr. 1, ETHZentrum, CH-8092 Zürich, Switzerland. E-mail:brigitte.kudielka@ ifv.gess.ethz.ch stress. As concluded recently, reactivity and recovery appear to be two important strategies for characterizing individual differences in stress-related cardiovascular regulation and probably capture substantially different information (Gerin et al., 2000; Hocking Schuler \& O’Brien, 1997; Kamarck \& Lovallo, 2003; Schwartz et al., 2003). Also Linden, Earle, Gerin, and Christenfeld (1997) reasoned that the study of recovery is a logical consequence of theories on stress-disease links.

As for clinical importance, Manuck, Kaplan, and Clarkson (1983) showed that elevated heart rate responses during a common laboratory stressor in cynomolgus monkeys was associated with heightened atherosclerosis in the coronary arteries, which is suggestive of a link between heart rate responses and cardiovascular disease. Also in humans, pronounced cardiovascular stress reactivity (Krantz \& Manuck, 1984; Lovallo \& Gerin, 2003; Schwarzt et al., 2003; Treiber et al., 2003) and recovery (Hocking Schuler \& O'Brien, 1997) may be an important behavioral mechanism underlying the pathophysiology of coronary artery disease (CAD). For example, heart rate elevations over time can lead to elevated tonic blood pressure lev- 
els and promote the development of CAD. However, it has to be taken into consideration that there is still a critical and ongoing debate about the adequacy of the so-called reactivity hypothesis (Linden, Gerin, \& Davidson, 2003; Pickering \& Gerin, 1990).

In the past, most studies focused separately on the effects of age or gender and only few data are available on gender differences of stress-induced heart rate responses in multiple age groups (e.g., including children; cf. Matthews \& Stoney, 1988). In addition, poststress recovery was suggested to serve as a marker for later onset of behavioral and biological disorders, but until now most studies did not report on poststress recovery processes. Because of the limited data sets available in the literature, a reanalysis of five different studies covering a wide age range was performed to elucidate differences in heart rate responsivity and recovery in men and women exposed to the TSST.

\section{Methods}

\section{Participants}

Data came from five independent studies conducted in the same laboratory by Buske-Kirschbaum et al. (unpublished data, 1997, 2003; children), Kirschbaum, Kudielka, Gaab, Schommer, and Hellhammer (1999; young adults), and Kudielka, Schmidt-Reinwald, Hellhammer, and Kirschbaum (1999, 2000; older adults). Eligibility was double checked in a brief medical examination (first laboratory appointment; exclusion criteria: psychiatric, endocrine, cardiovascular, or other specific chronic diseases, intake of psychoactive drugs, $\beta$-blockers, estrogens, or glucocorticoids). Only healthy (no patient groups), placebo-treated participants and premenopausal women in the luteal phase were included. The total sample consisted of 28 children (mean age: 12.3 years \pm 0.3 ; 9-15 years), 34 younger adults (mean age: 23.3 years \pm 0.5 ; $19-32$ years), and 26 elderly adults (mean age: 66.9 years \pm $1.1 ; 60-76$ years). Within each age group, men and women did not differ in chronological age (all $F$ 's $<1$ not significant, n.s.). The study protocols were approved by the local ethics committee. Adult participants and parents of all children gave written informed consent.

\section{Study Protocol}

At the second appointment (between 3 p.m. to 7 p.m.), participants were confronted with the Trier Social Stress Test (TSST). After arrival, participants were equipped with the Sport-Tester-Profi (Polar-Instruments, Gross-Gerau, Germany) for wireless transmission of heart rates with electrocardiogram precision. In adults, an intravenous catheter was inserted to obtain blood samples before and after the stress task for adrenocorticotropic hormone and cortisol assays, as reported elsewhere (Kudielka et al., 1999, 2000). After a rest period of at least $45 \mathrm{~min}$, all participants were confronted with the TSST.

In a recent meta-analysis, the TSST was found to provoke the most robust physiological stress responses as compared with several other stress tasks (Dickerson $\&$ Kemeny, 2002). For adults, the TSST protocol consists of a 3-min preparation period, a 5-min free speech, and a 5-min mental arithmetic task in front of an audience (Kirschbaum, Pirke, \& Hellhammer, 1993). The adapted TSST for children consists of a 5-min preparation period, 5 -min public speaking, and a 5-min mental arithmetic task. In the speaking task, children receive the beginning of a story and are prompted to complete the story as exciting as possible in front of the committee. In addition, the difficulty level of the mental arithmetic task (number subtraction) is adjusted for children (Buske-Kirschbaum et al., 1997).

\section{Heart Rate}

Mean heart rate responses were available for a prestress period of 5-min duration; the task preparation period (adults: $3 \mathrm{~min}$, children: $5 \mathrm{~min}$ ); and the speech task (5 min), the mental arithmetic (5 min), and a poststress period of 5-min duration. Heart rate values within a period were averaged to generate means.

\section{Visual Analogue Scales (VAS)}

VAS were used in adults to measure subjective perceptions of the stressor. In elderly participants, 14 VAS were applied measuring personal involvement; how strenuous, difficult, new, stressful, uncontrollable, and threatening the task was; and whether participants anticipated negative consequences. In younger adults, 6 VAS were applied assessing personal involvement; how stressful, new, uncontrollable, and unpredictable the task was; and whether the participant anticipated negative consequences. In the children samples no VAS were available.

\section{Statistical Analyses}

Analyses of variance (ANOVAs) were used to analyze basal heart rates and responses to the stressor. The statistical procedure was to first conduct an overall three-way ANOVA with the between-subjects factors age and gender and the repeated measure mean heart rates to consider all factors simultaneously. Post hoc, planned comparisons were applied for significant interactional effects where indicated. Two-way ANOVAs were conducted to describe gender effects for each age group separately. The parameter increase 
was defined as difference between the period with the highest individual heart rate and the individual prestressor baseline. The area-under-the-curve (AUC) was computed following the trapezoid formula. To investigate heart rate rebound and recovery after stress exposure the difference between prestress and poststress values (= rebound) and the difference between the period with the highest individual heart rate during the stress task and the individual poststress values (= recovery) were computed. All reported results were corrected by Greenhouse-Geisser procedure where appropriate, indicated by uneven degrees of freedom. Correlations were performed following the Pearson product-moment procedure. The significance level was set at $\alpha=.05$. All results shown are the mean \pm standard error of mean (SEM).

\section{Results}

\section{Prestress Baseline}

ANOVA results showed that baselines differed significantly between age groups but did not reveal a gender effect, age: $F(2,82)=7.79 p<.0008$; all other $F^{\prime}<$ 1 n.s.; see Figure 1. This indicates that children had much higher prestressor heart rates than adults (post hoc tests: children vs. younger adults $p=.001$, children vs. older adults $p=.0008$, younger adults vs. older adults $p=.70$ ). A significant negative correlation between prestressor heart rates and chronological age confirmed this observation $(r=-0.27, p=.01)$.

\section{Stress Response}

The overall three-way ANOVA resulted in a significant three-way interaction Gender $\times$ Age $\times$ Time, $F(4.7,193.1)=2.43 p=.04$, and significant two-way interactions Gender $\times$ Time, $F(2.4,193.1)=4.56, p<$ .008 , and Age $\times$ Time, $F(4.7,193.1)=5.06, p<.0003$,

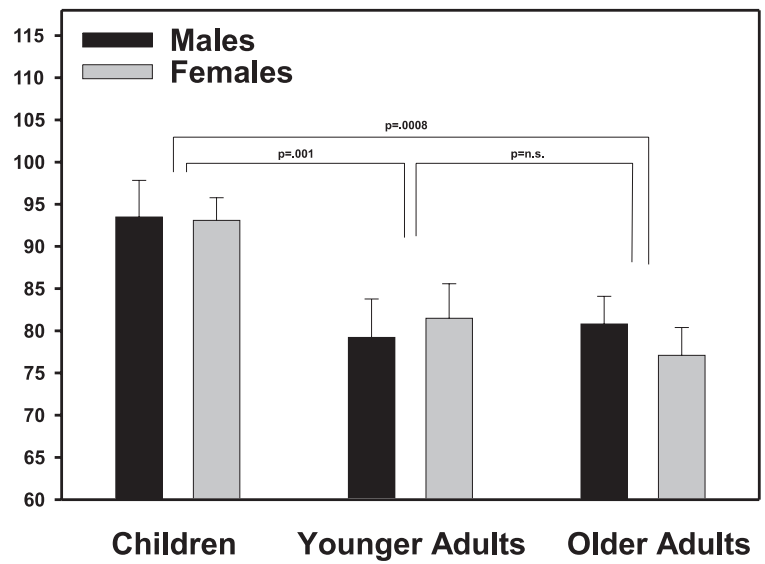

Figure 1. Prestressor baseline heart rates $( \pm S E M)$ in children, younger adults, and older adults. and significant main effects of age, gender, and time, age: $F(2,82=10.1, p<.0001$; gender: $F(1,82)=4.78$, $p<.03$; time: $F(2.4,193.1)=51.1, p<.0001$; see Figure 2. More details are reported in the following sections.

(A)

\section{Children}

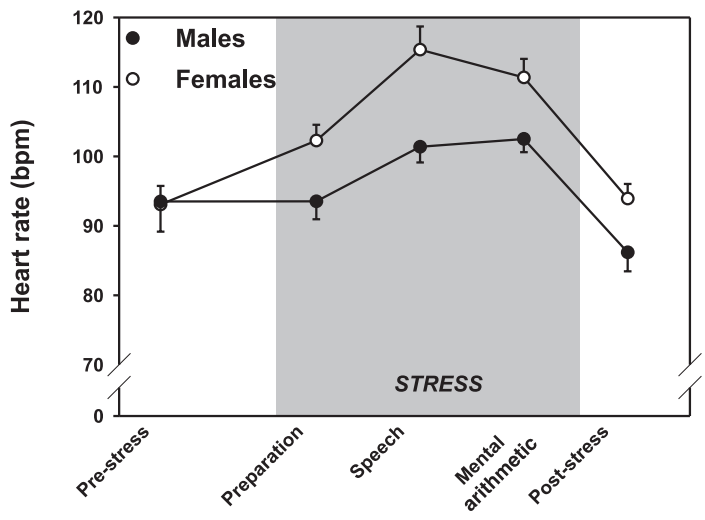

(B)

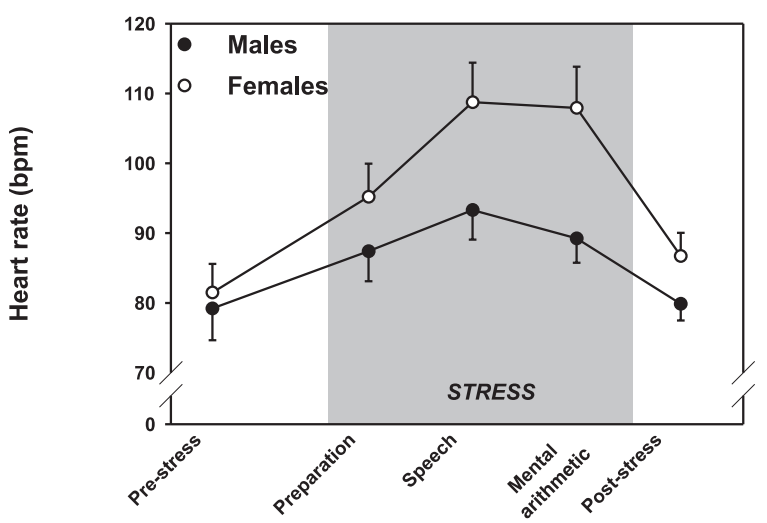

(C)

Older Adults

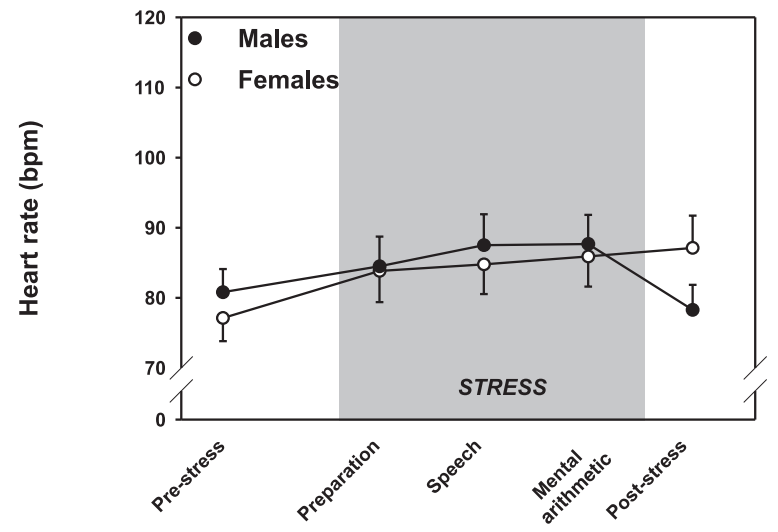

Figure 2. Heart rate response patterns in children (A), younger adults (B), and older adults (C) before (prestress), during (preparation, speech, mental, arithmetic), and after (poststress) confrontation with the TSST; the shaded areas indicated the duration of the TSST. 


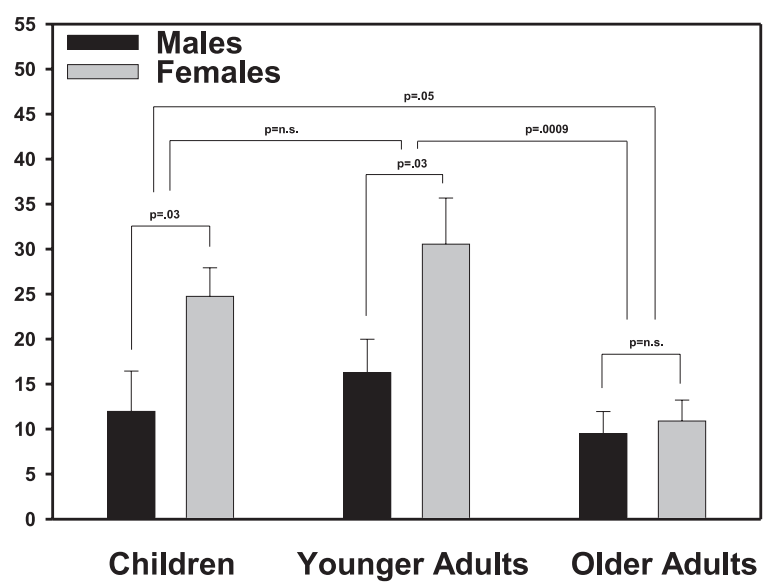

Figure 3. Heart rate increases $( \pm S E M)$ during the stress exposure in children, younger adults, and older adults.

Stress-induced increases showed significant main effects of age and gender, age: $F(2,82)=5.93, p<$ .004 ; gender: $F(1,82)=9.00, p<.004$; interaction: $F(2,82)=1.59, p<.2$, pointing at age and gender differences in heart rate responses to the TSST (post hoc tests: children vs. younger adults $p=.20$, children vs. older adults $p=.05$, younger vs. older adults $p=.0009$; see Figure 3). There was also a significant Age $\times$ Gender interaction for recovery, $F(2,82)=6.56, p<.002$, and a significant Gender $\times$ Time interaction for rebound, $F(1,82)=10.4, p=.002$. More details are reported in the following.

Chronological age correlated significantly with heart rate increases during stress $(r=-0.28, p=.009)$. However, a two-way analysis of covariance (ANCOVA) with chronological age as a covariate also resulted in a significant interaction Gender $\times$ Time, $F(2.4,207.9)=4.53, p<.008$, and a significant main effect of gender, $F(1,85)=5.63, p<.02$.

\section{Children}

A 2 (gender) $\times 5$ (time) ANOVA resulted in significant main effects of time and gender and a significant interaction Time $\times$ Gender, time: $F(4,104)=29.3, p<$ .0001 ; gender: $F(1,26)=7.43, p<.01$; interaction: $F(2.4,61.8)=3.00, p<.05$ (see Figure 2A). Furthermore, girls showed significantly larger heart rate increases, $F(1,26)=5.40, p<.03$, and a greater AUC, $F(1,26)=7.04, p<.01$, compared with boys, but no difference emerged for recovery, $F(1,26)=1.33, p<$ .30. A comparison between pretest and poststressor heart rates resulted in a significant interaction of Gender $\times$ Time, $F(1,26)=6.48, p<.02$. Planned comparisons confirmed that the poststressor heart rates were lower in boys than in girls $(p=0.03)$ and poststress heart rates compared to prestress baselines were lower in boys ( $p=0.003)$ but not in girls. In sum, these results point at a differential heart rate responsivity to the
TSST in children with girls showing higher stress responses than boys and only boys showing a recovery rebound (poststress heart rates are lower than prestress heart rates).

\section{Younger Adults}

The two-way ANOVA resulted in a significant main effect of time and significant interaction Time $\times$ Gender, time: $F(4,128)=24.1 p<.0001$; gender: $F(1,32)$ $=3.89, p<.06$; interaction: $F(1.9,61.0)=3.26, p<.05$ (see Figure 2B). Increase and recovery also differed significantly between men and women, increase: $F(1$, $32)=5.28, p<.03$; recovery: $F(1,32)=4.27, p<.05$; AUC: $F(1,32)=3.83, p<.06$, with women having higher responses. Poststressor baselines tended to be elevated in women, $F(1,32)=2.87, p<.10$, but there was no difference in rebound (all $F$ 's $<1.2$ n.s.). Finally, none of the six VAS correlated significantly with heart rate increase nor recovery (all $t$ 's $<1.9$ n.s.).

\section{Older Adults}

ANOVA results showed a significant main effect of time and Gender $\times$ Time interaction but no significant main effect of gender, time: $F(4,96)=8.33, p<.0001$; interaction: $F(3.0,72.5)=5.45, p<.002$; gender: $F<$ .01 n.s. (see Figure 2C). Neither stress-induced heart rate increases nor AUC differed between men and women, both $F \mathrm{~s}<1.8 n s$, but gender differences in recovery and rebound were highly significant, recovery: $F(1,24)=13.3, p<.001$; rebound interaction: $F(1,24)$ $=10.3, p<.004$, revealing that only men returned to prestressor values during the poststress period, whereas women still showed a continuous heart rate increase. Again, none of the 14 VAS correlated significantly with heart rate increase nor recovery (all $t \mathrm{~s}<$ $1.7, n s$ ), indicating that heart rate responses and recovery again were not associated with subjective ratings of the stressfulness of the task.

\section{Discussion}

The purpose of this report was to describe age and gender differences in stress-induced heart rate responses in a wide age range (9-76 years) and to include the assessment of poststress recovery as a part of the psychophysiological response to acute stress.

It is well known that heart rate regulation changes significantly during development, which is most obvious in higher basal heart rates in infants and children compared to adults (Tanaka, Monahan, \& Seals, 2001). In accordance, our data showed a negative correlation between basal heart rates and chronological age. In response to the TSST, the data revealed significantly lower heart rate responses in older participants com- 
pared to younger adults and children, which is in line with most earlier human studies (Boutcher \& Stocker, 1996; Furchtgott \& Busemeyer, 1979; Gintner, Hollandsworth, \& Intrieri, 1986), although other studies did not observe the expected age effects (Saab, Matthews, Stoney, \& McDonald, 1989; Steptoe, Moses, \& Edwards, 1990). Those inconsistencies might be explained by psychological factors, at least in part. Uchino, Kiecolt-Glaser, and Cacioppo (1992) reported that caregivers with high social support displayed the typical age-related decreases in heart rate reactivity, whereas caregivers low in social support displayed age-related increases in their heart rate stress responses. Mechanisms underlying the observed age-specific effects include structural changes; decreased muscle mass of the heart and contractability; and alterations in the sensitivity of baroreceptors, neurotransmission, and adrenergic receptors (Lakatta, 1993; Schocken \& Roth, 1977).

The finding that women had larger heart rate responses to the TSST than men seem to fit to a conclusion drawn earlier that women are more likely to be cardiac reactors, whereas men are more prone to be vascular reactors (Allen, Stoney, Owens, \& Matthews, 1993). It can be speculated that underlying mechanisms might operate at the receptor level (density and sensitivity) and/or through alterations in parasympathetic control of the heart and/or structural differences in the myocardium (e.g., gender difference exist in the size of the heart, muscle mass, and ventricular volume). However, this gender difference did not emerge in the older adults. In an early review, Polefrone and Manuck (1987) also concluded that "females have typically (but not always) shown larger heart rate reactions than males" (p. 24). Potential modulators of different heart rate responses in premenopausal versus postmenopausal women are the reproductive hormones. However, reported effects concerning menopausal status remained inconsistent (Saab et al., 1989; Steptoe, Fieldman, Evans, \& Perry, 1996; Steptoe et al., 1990). Furthermore, it can be hypothesized that gender differences are task dependent. It was shown that women had larger heart rate responses to different speech and math tasks but not during mirror-imaging tracing and real-life examinations (Anishchenko, Igosheva, Yakusheva, Glushkovskaya-Semyachkina, \& Khokhlova, 2001; Girdler, Turner, Sherwood, \& Light, 1990; Tersman, Collins \& Eneroth, 1991). This appears to fit to the observation that men outperformed women in serial subtraction, whereas women showed higher performance in mirror-imaging tracing (Matthews \& Stoney, 1988). However, no significant relation could be found between subjective stress ratings and the physiological stress responses in our data (performance data was not available).

Concerning recovery, Watanabe, Thamilarasan, Blackstone, Thomas, \& Lauer (2001) recently showed that an attenuated heart rate recovery after physical ex- ercise was predictive of the mortality rate, and Imai et al. (1994) found a significant correlation between heart rate recovery after physical exercise and functional capacity. Our data show that heart rate recovery patterns differed as a function of age and gender. Recently, Earle, Linden, and Weinberg (1999) investigated heart rate recovery after a mental arithmetic task with and without harassment in young adults. They reported a faster heart rate recovery in women compared to men in the 1st min of the recovery period after psychological stress. However, this effect did not remain significant throughout the recovery period.

Possible clinical implications of these findings remain to be elucidated in future studies, especially considering possible limitations of our data set. Although each of the five cohorts was exposed to the highly standardized TSST protocol, some (in most parts inevitable) differences between the studies could not be avoided (e.g., seasonal differences, personnel involved; adapted TSST version for children; possible group differences in fitness or psychological factors). Another important issue of laboratory stress studies is their ecological validity. In a recent review, Kamarck and Lovallo (2003) remind that more research is warranted to proof lab-to-life generalizability to evaluate emerging evidence of the relation between cardiovascular reactivity measures and disease risk. In sum, in addition to maximum stress responses, upcoming laboratory and clinical studies should include more fine-grained investigations of the recovery phase to be able to investigate possible consequences of altered cardiovascular recovery processes and associations between recovery patterns and disease risk.

\section{References}

Allen, M. T., Stoney, C. M., Owens, J. F., \& Matthews, K. A. (1993). Hemodynamic adjustments to laboratory stress: the influence of gender and personality. Psychosomatic Medicine, 55, 505-517.

Anishchenko, T., Igosheva, N., Yakusheva, T., GlushkovskayaSemyachkina, O., \& Khokhlova, O. (2001). Normalized entropy applied to the analysis of interindividual and gender-related differences in the cardiovascular effects of stress. European Journal of Applied Physiology, 85, 287-298.

Boutcher, S. H., \& Stocker, D. (1996). Cardiovascular response of young and older males to mental challenge. Journal of Gerontology, B51, 261-267.

Buske-Kirschbaum, A., Jobst, S., Wustmans, A., Kirschbaum, C., Rauh, W., \& Hellhammer, D. (1997). Attenuated free cortisol response to psychosocial stress in children with atopic dermatitis. Psychosomatic Medicine, 59, 419-426.

Buske-Kirschbaum, A., von Auer, K., Krieger, S., Weis, S., Rauh, W., Hellhammer, D. (2003). Blunted cortisol responses to psychosocial stress in asthmatic children: A general feature of atopic disease? Psycholsomatic Medicine, 65, 806-810.

Dickerson, S. S., \& Kemeny, M. E. (2004). Acute stressors and cortisol responses: A theorical integration and synthesis of laboratory research. Psychological Bulletin, 130, 355-391. 
Earle, T. L., Linden, W., \& Weinberg, J. (1999). Differential effects of harassment on cardiovascular and salivary cortisol stress reactivity and recovery in women and men. Journal of Psychosomatic Research, 46, 125-141.

Furchtgott, E., \& Busemeyer, J. K. (1979). Heart rate and skin conductance during cognitive processes as a function of age. Journal of Gerontology, 34, 183-190.

Gerin, W., Pickering, T. G., Glynn, L., Christenfeld, N., Schwartz, A., Carroll, D., et al. (2000). An historical context for behavioral models of hypertension. Journal of Psychosomatic Research, 48, 369-377.

Gintner, G. G., Hollandsworth, J. G., \& Intrieri, R. C. (1986). Age differences in cardiovascular reactivity under active coping conditions. Psychophysiology, 23, 113-120.

Girdler, S. S., Turner, J. R., Sherwood, A., \& Light, K. C. (1990). Gender differences in blood pressure control during a variety of behavioral stressors. Psychosomatic Medicine, 52, 571-591.

Haynes, S. N., Gannon, L. R., Orimoto, L., O’Brien, W. H., \& Brandt, M. (1991). Psychophysiological assessment of poststress recovery. Psychological Assessment: A Journal of Consulting and Clinical Psychology, 3, 356-365.

Hocking Schuler, J. L., \& O’Brien, W. H. (1997). Cardiovascular recovery from stress and hypertension risk factors: A meta-analytic review. Psychophysiology, 34, 649-659.

Imai, K., Sato, H., Hori, M., Kusuoka, H., Ozaki, H., Yokoyama, H., et al. (1994). Vagally mediated heart rate recovery after exercise is accelerated in athletes but blunted in patients with chronic heart failure. Journal of the American College of Cardiology, 24, 1529-1535.

Kamarck, T. W., \& Lovallo, W. R. (2003). Cardiovascular reactivity to psychological challenges. Conceptual and measurement considerations. Psychosomatic Medicine, 65, 9-21.

Kirschbaum, C., Kudielka, B. M., Gaab, J., Schommer, N. C., \& Hellhammer, D. H. (1999). Impact of gender, menstrual cycle phase, and oral contraceptives on the activity of the hypothalamus-pituitary-adrenal axis. Psychosomatic Medicine, 61, 154-162.

Kirschbaum, C., Pirke, K.-M., \& Hellhammer, D. H. (1993). The 'Trier Social Stress Test'-a tool for investigating psychobiological stress responses in a laboratory setting. Neuropsychobiology, 28, 76-81.

Krantz, D. S., \& Manuck, S. B. (1984). Acute psychophysiological reactivity and risk of cardiovascular disease: A review and methodologic critique. Psychological Bulletin, 96, 435-464.

Kudielka, B. M., Schmidt-Reinwald, A. K., Hellhammer D. H., \& Kirschbaum C. (1999). Psychological and endocrine responses to psychosocial stress and Dex-CRF in healthy postmenopausal women and young controls: The impact of age and a two-week estradiol treatment. Neuroendocrinology, 70, 422-430.

Kudielka, B. M., Schmidt-Reinwald, A. K., Hellhammer, D. H., Kirschbaum C. (2000). Psychosocial stress and functioning of the hypothalamic-pituitary-adrenal axis: No evidence for a reduced resilience in elderly men. Stress, 3, 229-240.

Lakatta, E. G. (1993). Cardiovascular regulatory mechanisms in advanced age. Physiological Reviews, 73, 413-467.

Linden, W., Earle, T. L., Gerin, W., \& Christenfeld, N. (1997). Physiological stress reactivity and recovery: Conceptual siblings separated at birth? Journal of Psychosomatic Research, 42, $117-135$.
Linden, W., Gerin, W., \& Davidson, K. (2003). Cardiovascular reactivity: Status quo and a research agenda fort he new millennium. Psychosomatic Medicine, 65, 5-8.

Lovallo, W. R., \& Gerin, W. (2003). Psychophysiological reactivity: Mechanisms and pathways to cardiovascular disease. Psychosomatic Medicine, 65, 36-45.

Manuck, S. B., Kaplan, J. R., \& Clarkson, T. B. (1983). Behaviorally induced heart rate reactivity and atherosclerosis in cynomolgus monkeys. Psychosomatic Medicine, 45, 95-108.

Matthews, K. A., Salomon, K., Brady, S. S., Allen, M. T. (2003). Cardiovascular reactivity to stress predicts future blood pressure in adolescence. Psychosomatic Medicine, 65, 410-415.

Matthews, K. A., \& Stoney, C. M. (1988). Influences of sex and age on cardiovascular responses during stress. Psychosomatic Medicine, 50, 46-56.

Pickering, T. G., \& Gerin, W. (1990). Cardiovascular reactivity in the laboratory and the role of behavioral factors in hypertension. A critical review. Annals of Behavioral Medicine, 12, 3-16.

Polefrone, J. M., \& Manuck, S. B. (1987). Gender differences in cardiovascular and neuroendocrine response to stress. In R. S. Barnett, L. Biener \& G. K. Baruch (Eds.), Gender and stress (pp. 13-38). New York: Free Press.

Saab, P. G., Matthews, K. A., Stoney, C. M., \& McDonald, R. H. (1989). Premenopausal women differ in their cardiovascular and neuroendocrine responses to behavioral stressors. Psychophysiology, 26, 270-280.

Schocken, D. D., \& Roth, G. S. (1977). Reduced beta-adrenergic receptor concentrations in ageing man. Nature, 267, 856-858.

Schwartz, A. R., Gerin, W., Davidson, K. W., Pickering, T. G., Brosshot, J. F., Thayer, J. F., et al. (2003). Toward a causal model of cardiovascular responses to stress and the development of cardiovascular disease. Psychosomatic Medicine, 65, 22-35.

Seeman, T. E., \& Robbins, R. J. (1994). Aging and hypothalamic-pituitary-adrenal response to challenge in humans. Endocrine Reviews, 15, 233-260.

Steptoe, A., Fieldman, G., Evans, O., \& Perry, L. (1996). Cardiovascular risk and responsivity to mental stress: The influence of age, gender and risk factors. Journal of Cardiovascular Risk, 3, 83-93.

Steptoe, A., Moses, J., \& Edwards, S. (1990). Age-related differences in cardiovascular reactions to mental stress tests in women. Health Psychology, 9, 18-34.

Tanaka, H., Monahan, K. D., \& Seals, D. R. (2001). Age-predicted maximal heart rate revisited. Journal of the American College of Cardiology, 37, 153-156.

Tersman, Z., Collins, A., \& Eneroth, P. (1991). Cardiovascular responses to psychological and physiological stressors during the menstrual cycle. Psychosomatic Medicine, 53, 185-197.

Treiber, F. A., Kamarck, T., Schneiderman, N., Sheffield, D., Kapuku, G., \& Taylor, T. (2003). Cardiovascular reactivity and development of preclinical and clinical disease states. Psychosomatic Medicine, 65, 46-62.

Uchino, B. N., Kiecolt-Glaser, J. K., \& Cacioppo, J. T. (1992). Age-related changes in cardiovascular responses as a function of a chronic stressor and social support. Journal of Personality and Social Psychology, 63, 839-846.

Watanabe, J., Thamilarasan, M., Blackstone, E. H., Thomas, J. D, \& Lauer, M. S. (2001). Heart rate recovery immediately after treadmill exercise and left ventricular systolic dysfunction as predictors of mortality. Circulation, 104, 1911-1916. 